Forschende

Komplementärmedizin unc Klassische Naturheilkunde

\section{Tagungen und Kongresse - Meetings and Conferences}

Forsch Komplementärmed Klass Naturheilkd 2003;10:290

\subsection{1 .2003}

München

Deutschland

\subsection{1.-09.11.2003}

Bremen

Deutschland

\subsection{1.-11.11.2003}

Wiesbaden

Deutschland

\subsubsection{3 \\ Baden}

Schweiz

\subsection{1.-06.12.2003} Puerto de la Cruz

Teneriffa
Viel genutzt, zu wenig erforscht - Zukunft der Naturheilverfahren/ Komplementärmedizin in den Universitäten

\section{Bremer-Chiropraktik-Seminar}

Thema: LWS-Becken

\section{Echokardiographie-Aufbaukurs}

18. Schweizerische Tagung für Phytotherapie - Phytotherapie und Stoffwechsel

26. Internationaler interdisziplinärer Seminarkongress für ärztliche Fortbildung in Puerto de la Cruz / Teneriffa

Deutscher Kongress für integrative Onkologie Ein interdisziplinärer onkologischer Kongress

Ultraschall-Echokardiographie-Refresherkurs, Teil 1
Auskunft: Zentrum für naturheilkundliche

Forschung

II. Med. Klinik und Poliklinik

TU München

Kaiserstr. 9

D-80801 München

Fax +4989393484

www.muemo.med.tu-muenchen.de

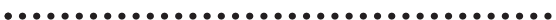

Auskunft: Deutsch-amerikanische Gesellschaft

für Chiropraktik e.V.

Parkallee 157

D-28209 Bremen

Tel. +49 421347 97-55, Fax -62

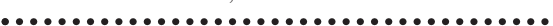

Auskunft: Berufsverband Deutscher Internisten e.V.

Kongressabteilung

Postfach 1566

D-65005 Wiesbaden

Tel. +49 611 18133-22 oder -24, Fax -23

E-mail info@bdi.de

www.bdi.de

Auskunft: Sekretariat SMGP

Keltenstr. 40

CH-8044 Zürich

Tel. +41 125 21-879, Fax -906

E-mail sekretariat-smgp@swissonline.ch

www.smgp.ch

Auskunft: Berufsverband Deutscher Internisten e.V. Kongressabteilung

Postfach 1566

D-65005 Wiesbaden

Tel. +49 611 18133-22 oder -24, Fax -23

E-mail info@bdi.de

www.bdi.de

Auskunft: gfmk KG

Kongresse

Postfach 250224

D-51324 Leverkusen

Tel. +49 214 31057-0, Fax -19

E-mailinfo@gfmk.de

www.gfmk-kongresse.de

\section{Auskunft: $\mathrm{ZÄN}$}

Am Promenadenplatz

D-72250 Freudenstadt

Tel. + 497441 91858-0, Fax -22

E-mail ZAEN-Freudenstadt@t-online.de

www.zaen.org Auskunft: Berufsverband Deutscher Internisten e.V. Kongressabteilung

Postfach 1566

D-65005 Wiesbaden

Tel. +49 611 18133-22 oder -24, Fax -23

E-mailinfo@bdi.de

www.bdi.de 